\title{
Transverse-momentum resummation for gaugino-pair production at the LHC
}

\author{
Jonathan DEBOVE* \\ Laboratoire de Physique Subatomique et de Cosmologie, Université Joseph \\ Fourier/CNRS-IN2P3/INPG, 53 Avenue des Martyrs, F-38026 Grenoble, France \\ E-mail: deboved lpsc.in2p3.fr
}

\begin{abstract}
We present a first precision analysis of the transverse-momentum spectrum of gaugino pairs produced at the LHC with center-of-mass energies of 10 or $14 \mathrm{TeV}$. Our calculation is based on a universal resummation formalism at next-to-leading logarithmic accuracy, which is consistently matched to the perturbative prediction at $O\left(\alpha_{s}\right)$. Numerical results are given for the "gold-plated" associated production of neutralinos and charginos for a typical benchmark point in the constrained Minimal Supersymmetric Standard Model. We show that the matched resummation results differ considerably from the Monte Carlo predictions employed traditionally in experimental analyses. We also investigate in detail the theoretical uncertainties coming from scale and parton-density function variations and non-perturbative effects.
\end{abstract}

European Physical Society Europhysics Conference on High Energy Physics

July 16-22, 2009

Krakow, Poland

\footnotetext{
* Speaker.
} 


\section{Introduction}

The Minimal Supersymmetric Standard Model (MSSM) is one of the most appealing extensions of the Standard Model of particle physics [1]. 2]. In the MSSM, the fermionic partners of the neutral (charged) gauge and Higgs bosons are called neutralinos (charginos) and are of particular importance. Stabilized by $R$-symmetry, the lightest neutralino is a very promising candidate for the dark matter observed in the universe. Moreover, the values of gaugino masses and mixings are key ingredients to understand both the electroweak symmetry and the supersymmetry breakings. Since these particles may be light enough to be produced at current hadron colliders, they have been the object of particular attention, and cross sections for the production of gaugino pairs have been extensively studied at leading order [3, $4,5,6$, 7 and next-to-leading order of perturbative QCD [8].

While particle pairs are produced with zero transverse momentum $\left(p_{T}\right)$ in the Born approximation, gluon bremsstrahlung induces non-zero $p_{T}$ at $O\left(\alpha_{s}\right)$ in the strong coupling constant. The aim of this work is to perform an accurate calculation of the $p_{T}$-distribution of the gaugino pairs. Although the use of the perturbative expansion in powers of $\alpha_{s}$ is fully justified, when the $p_{T}$ of the produced system is of the order of its invariant mass $M$, the convergence of the perturbative expansion is spoiled by powers of large logarithmic terms, $\ln M^{2} / p_{T}^{2}$, in the region where $p_{T} \ll M$. Therefore the enhanced logarithms must be resummed to all orders in $\alpha_{s}$.

\section{Transverse-momentum resummation}

The method to systematically perform all-order resummation of classes of enhanced logarithms is well-known [9, 10] and is performed in impact parameter $(b)$ space. Thus we work with the Fourier transform $W$ of the partonic cross section defined by

$$
\frac{d \sigma^{R E S}}{d M^{2} d p_{T}^{2}}(z)=\frac{z}{M^{2}} \int \frac{d^{2} b}{4 \pi} e^{i \mathbf{b} \cdot \mathbf{p}_{\mathbf{T}}} W\left(b^{2}, M^{2}, z\right),
$$

where $z=M^{2} / s$ and $\sqrt{s}$ is the partonic center-of-mass energy. The renormalisation scale $\left(\mu_{R}\right)$ and the factorisation scale $\left(\mu_{F}\right)$ dependencies have been removed for the sake of simplicity. After a Mellin transform with resptect to $z$, the $N$-moments of the $W$-function

$$
W\left(b^{2}, M^{2}, N\right)=H\left(M^{2}, N\right) \exp \left[G\left(b^{2}, N\right)\right]
$$

factorize into a $b$-independent function $H$ and an exponential form factor $\exp [G]$. The function $H$ encodes the full process dependence, and the form factor $\exp [G]$ resums all the terms that are order by order logarithmically divergent. The exponent $G$, which controls the soft gluon emmission, is thus universal, i.e. it does not depend on the final state of the process [10]. The general expressions for the $H$ - and $G$-functions can be found in Refs. [10, 11].

After the large logarithms have been resummed in $b$-space, we have to switch back to $p_{T}$-space in order to achieve a phenomenological study. Special attention has to be paid to the singularities in the exponent $G$. They are related to the presence of the Landau pole in the perturbative running of $\alpha_{s}$, and a prescription is needed. In our numerical study, we follow Ref. [12] and deform the integration contour in the complex $b$-plane. Note that the divergent behavior of $\alpha_{s}$ signals the 


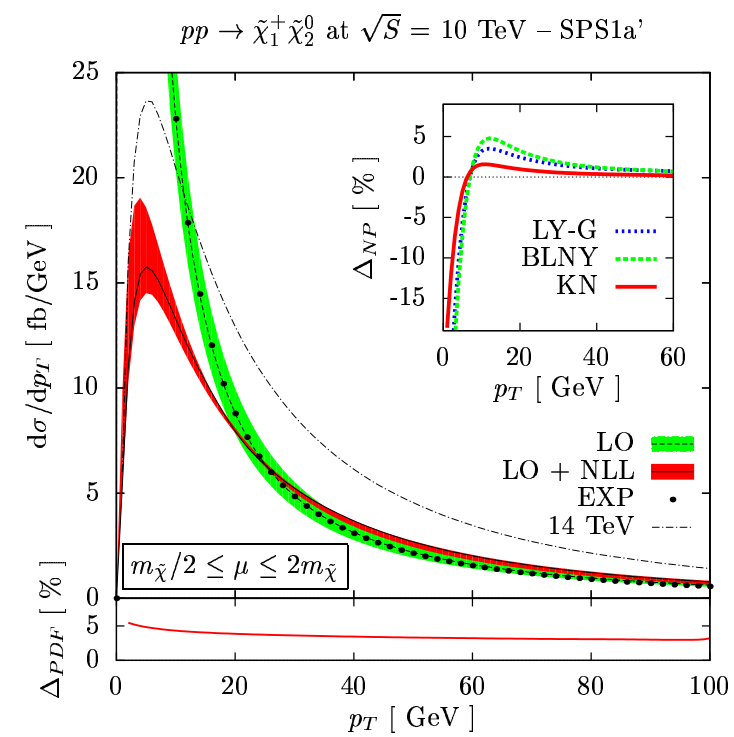

Figure 1: $p_{T}$-spectra of $\tilde{\chi}_{1}^{+} \tilde{\chi}_{2}^{0}$-pairs at the LHC. The LO calculation (dashed) is matched to the resummed calculation (full) by subtracting its fixed-order expansion (dotted). The scale (shaded band), PDF (below) and NP (insert) uncertainties are shown, as well as the matched result for the LHC design energy of $14 \mathrm{TeV}$ (dot-dashed).

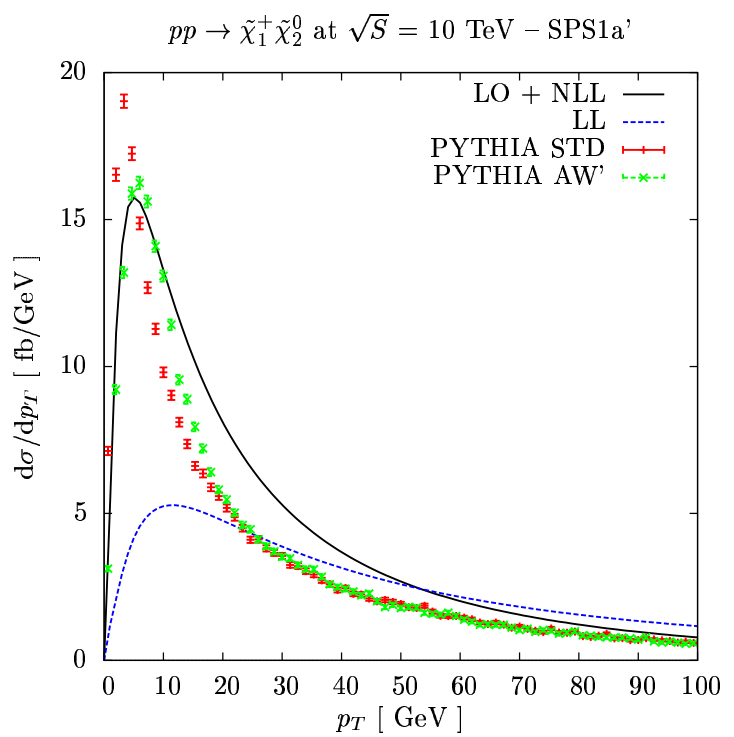

Figure 2: $p_{T}$-spectra of $\tilde{\chi}_{1}^{+} \tilde{\chi}_{2}^{0}$-pairs at the LHC. The matched LO+NLL (full) and the LL (dashed) results are compared with the predictions of the PYTHIA parton shower with default (bars) and tuned (crosses) parameters.

onset of non-perturbative (NP) effects at large $b$. These unknown effects may be extracted from experiment and can then be included in the exponential form factor.

Finally, in order to conserve the full information contained in the fixed-order calculation, the $O\left(\alpha_{s}\right)$ and the resummed calculations are matched by subtracting from their sum the resummed cross section truncated at $O\left(\alpha_{s}\right)$.

\section{Numerical results}

We now present numerical results for the associated production of neutralinos and charginos at the LHC with a hadronic center-of-mass energy of $\sqrt{S}=10$ and $14 \mathrm{TeV}$. Results for the pair production of neutralinos and charginos at the Tevatron and at the LHC can be found in Ref. [11]. The parton densities are evaluated in the most recent parametrisation of the CTEQ collaboration CTEQ 6. 6M [13] with $\mu_{F}$ (and $\mu_{R}$ ) set to the average mass $m_{\tilde{\chi}}$ of the final state particles, and $\alpha_{s}$ is evaluated at two-loop accuracy. In the following, we choose the minimal supergravity benchmark point SPS1a' [14] and obtain the weak-scale supersymmetric parameters through the computer code SuSpect 2.3 [15].

In Fig. 1, we show the $p_{T}$-spectra of chargino-neutralino pairs produced at the Tevatron and at the LHC. As expected, the $O\left(\alpha_{s}\right)$ calculation (LO) diverges at low $p_{T}$, but becomes finite after having been matched to the resummed prediction at next-to-leading logarithmic (LO+NLL) accuracy. In this region the perturbative expansion (EXP) of the resummed prediction coincides with the LO one. For comparison, the LO+NLL prediction for the $14 \mathrm{TeV}$ design energy of the LHC is also pre- 
sented. We also study three different sources of uncertainty: the scale variations evaluated in the range $\left[m_{\tilde{\chi}} / 2,2 m_{\tilde{\chi}}\right]$, the PDF uncertainties $\Delta_{P D F}$ as defined by the CTEQ collaboration [13] and the choice of three parametrisations for the NP form factor evaluated through $\Delta_{N P}=\left(d \sigma^{N P}-d \sigma\right) / d \sigma$ [16, 17]. The scale dependence of the LO+NLL prediction is clearly improved with respect to the LO result and the other uncertainties are all smaller than $5 \%$ for $p_{T}>5 \mathrm{GeV}$.

In Fig. 2, we compare our LO+NLL prediction with our resummed result at leading-logarithmic (LL) accuracy and two different setups for the PYTHIA 6 . $[$ [18] Monte Carlo (MC) generator. We see that the default (STD) MC simulation is clearly improved beyond the LL approximation and approaches the LO+NLL result, but peaks at slightly smaller values of $p_{T}$. This behavior can be improved by tuning the intrinsic $p_{T}$ of the partons in the hadron (AW') [11], but both simulations underestimate the intermediate $p_{T}$-region.

\section{Conclusion}

In summary, we have calculated the $p_{T}$-distribution of gaugino pairs produced at the LHC at next-to-leading logarithmic accuracy. This has the advantage of making the perturbative predictions finite and reducing the scale uncertainties. We have also compared our results with a MC generator commonly used for experimental analyses. All these features will possibly lead to improvements for the experimental determination of the gaugino parameters.

\section{References}

[1] H. P. Nilles, Phys. Rept. 110 (1984) 1.

[2] H. E. Haber and G. L. Kane, Phys. Rept. 117 (1985) 75.

[3] V. D. Barger, R. W. Robinett, W. Y. Keung and R. J. N. Phillips, Phys. Lett. B 131 (1983) 372.

[4] S. Dawson, E. Eichten and C. Quigg, Phys. Rev. D 31 (1985) 1581.

[5] G. Bozzi, B. Fuks, B. Herrmann and M. Klasen, Nucl. Phys. B 787 (2007) 1.

[6] J. Debove, B. Fuks and M. Klasen, Phys. Rev. D 78 (2008) 074020.

[7] B. Fuks, B. Herrmann and M. Klasen, Nucl. Phys. B 810 (2009) 266.

[8] W. Beenakker, M. Klasen, M. Krämer, T. Plehn, M. Spira and P. M. Zerwas, Phys. Rev. Lett. 83 (1999) 3780 [Erratum-ibid. 100 (2008) 029901].

[9] J. C. Collins, D. E. Soper and G. Sterman, Nucl. Phys. B 250 (1985) 199.

[10] G. Bozzi, S. Catani, D. de Florian and M. Grazzini, Nucl. Phys. B 737 (2006) 73.

[11] J. Debove, B. Fuks and M. Klasen, arXiv:0907.1105 [hep-ph].

[12] E. Laenen, G. Sterman and W. Vogelsang, Phys. Rev. Lett. 84 (2000) 4296.

[13] P. M. Nadolsky et al., Phys. Rev. D 78 (2008) 013004.

[14] J. A. Aguilar-Saavedra et al., Eur. Phys. J. C 46 (2006) 43.

[15] A. Djouadi, J. L. Kneur and G. Moultaka, Comput. Phys. Commun. 176 (2007) 426.

[16] F. Landry, R. Brock, P. M. Nadolsky and C. P. Yuan, Phys. Rev. D 67 (2003) 073016.

[17] A. V. Konychev and P. M. Nadolsky, Phys. Lett. B 633 (2006) 710.

[18] T. Sjöstrand, S. Mrenna and P. Skands, JHEP 0605 (2006) 026. 